\title{
The Determination of Glycopeptides by Liquid Chromatography/Mass Spectrometry with Collision-Induced Dissociation
}

\author{
James J. Conboy and Jack D. Henion \\ Drug Testing and Toxicology, New York State College of Veterinary Medicine, \\ Cornell University, Ithaca, New York, USA
}

\begin{abstract}
Glycopeptides derived from ribonuclease B and ovomucoid have been subjected to collisioninduced dissociation (CID) in the second quadrupole of a triple quadrupole mass spectrometer. Doubly charged parent ions gave predictable fragmentation that yielded partial sequence information of the attached oligosaccharide as Hex and HexNAc units. Common oxonium ions are observed in the product ion mass spectra of the glycopeptides that correspond to HexNAc ${ }^{+}(m / z 204)$ and HexHexNAc ${ }^{+}(m / z 366)$. A strategy for locating the glycopeptides in the proteolytic digest mixtures of glycoproteins by ions spray liquid chromatography mass spectrometry (LC/MS) is described by utilizing CID in the declustering region of the atmospheric pressure ionization mass spectrometer to produce these characteristic oxonium ions. This LC/CID/MS approach is used to identify glycopeptides in proteolytic digest mixtures of ovomucoid, asialofetuin, and fetuin. LC/CID/MS in the selected ion monitoring mode may be used to identify putative glycopeptides from the protenlytir digest of fetuin. (f Am Soc Mass Spectrom 1992, 3, 804-814)
\end{abstract}

A mong the most challenging structural problems arising from posttranslational modification of proteins is the determination of glycosylation sites and glycan structure of glycoproteins [1]. The biological synthesis and processing of glycoprotein carbohydrates result in microheterogeneity due to heterogeneous populations of structurally related oligosaccharides and site heterogeneity due to different populations of attached carbohydrate at different glycosyation sites [2]. The carbohydrate moieties have been shown to influence activity, clearance from circulation, targeting to particular tissues, solubility, and stability of glycoproteins [3]. Glycoproteins have been characterized by lectin affinity chromatography [4], exoglycosidase digestion [5], NMR spectroscopy [6], and mass spectrometry $[7,8]$.

Fast-atom bombardment mass spectrometry (FAB/MS) [9] has been utilized extensively for the characterization of oligosaccharides released from glycoproteins by either chemical or enzymatic procedures $[10,11]$. Reductive amination procedures have been developed with aminobenzoic acid esters (ABEE) to direct mass spectral fragmentation and to add a lipophilic UV-absorbing chromophore for high-performance liquid chromatographic (HPLC) separation and detection of oligosaccharide mixtures [12], and highenergy collision-induced dissociation (CID) tandem

Address reprint requests to Jack D. Henion, Drug Testing and Toxicology, New York State College of Veterinary Medicine, Cornell University, 925 Warren Drive, Ithaca, NY 14850 mass spectrometry (MS/MS) in the negative ion mode of the deprotonated ABEE-derivatized molecules yields useful interpretative information [13]. However, these and other FAB/MS strategies [14-17] do not entirely satisfy the stringent demands of glycoprotein characterization [17].

Improved mass spectrometric methods to characterize oligosaccharides attached to glyproteins and to screen for glycosylated peptides in proteolytic digest mixtures of glycoproteins are needed. Electrospray ionization [18] offers the advantage that underivatized biomolecules, including proteins and peptides, may be desorbed as ions from the condensed phase into the gas phase under very mild ionization conditions. The mechanism of this ion evaporation [19] provides an electrical driving force that desorbs both hydrophilic and hydrophobic peptides from aqueous solvents. The preferential desorption of hydrophobic species due to increased surface activity in the matrix observed with $\mathrm{FAB} / \mathrm{MS}$ [20] is not seen with electrospray. $\mathrm{Hy}-$ drophilic, nonionic species such as polyether ionophores [21] and N-linked oligosaccharides [22] may be desorbed as ammonium or alkali metal adducts by electrospray ionization.

A preferred covalent derivative for a nonionic biopolymer such as an oligosaccharide would be one that contains a charge or could assume a charge in solution. Since glycoproteins contain carbohydrates that are either N-linked to asparagine or O-linked to serine or threonine [17], the protein or peptides could serve as a logical ionization site or "derivative" for the 
attached oligosaccharide. Intact glycoproteins have been studied by electrospray [23], although the multiply charged nature of observed ions can place a high demand on mass resolution of the quadrupole analyzer used such that a deconvolution algorithm may be required to determine the glycoforms [24].

Electrospray ionization also offers advantages for sequence determination of biopolymers by mass spectrometry. The increased kinetic energy of multiply charged precursor ions combined with internal energy due to charge repulsion causes abundant fragmentation of multiply charged peptides in the collision cell of a triple quadrupole mass spectrometer [25, 26]. A considerable advantage of electrospray over other mass spectrometric ionization techniques to determine peptides in proteolytic digest mixtures of proteins is the relative ease of interfacing with reversed-phase HPLC capability [27]. Ion spray LC/MS [28] has been used in conjunction with reversed-phase HPLC gradients containing trifluoroacetic acid to determine peptides with microbore [29] and packed-capillary [30] column separations.

The purpose of this work is to develop methodology for the selective determination of glycopeptides in proteolytic digests of glycoproteins by CID and LC/MS. Model N-linked tryptic or tryptic-like glycopeptides (C-terminal Arg or Lys) derived from ribonuclease B (RNase B) and ovomucoid have been studied by tandem mass spectrometry and the fragmentation patterns in the resulting mass spectra have been interpreted. The structural information obtained was evaluated with respect to the partial sequencing of the oligosaccharide portion and the molecular weight determination of the peptide portion. Characteristic oxonium ions resulting from the carbohydrate portion that are common to the different glycopeptides were identified. An LC/MS strategy was then developed to locate glycopeptides in the enzymatic digest mixtures of glycoproteins by CID in the declustering region of the atmospheric pressure ionization mass spectrometer [31] to form these characteristic oxonium ions. This approach, which will be referred to as LC/CID/MS, was demonstrated to screen for glycopeptidesin the proteolytic digests of the glycoproteins ovomucoid, asialofetuin, and fetuin.

\section{Experimental}

\section{Chemicals}

RNase B was purchased from Sigma Chemical Co. (St. Louis, MO), and purified by cation exchange chromatography to remove nonglycosylated (possibly deamidated) RNase A. Ovomucoid, fetuin, and asialofetuin (ASF) were purchased from Sigma Chemical Co. and used without any prior purification. Dithiothreitol (DTT), $8 \mathrm{M}$ guanidine hydrochloride and trifluoroacetic acid (TFA) were purchased from the Pierce Chemical Co. (Rockford, IL). Iodoacetic acid, ammonium bicarbonate, tris(hydroxymethyl)- aminomethane (tris) buffer, $96 \%$ formic acid, L-1tosylamide-2-phenylethyl chloromethyl ketone-treated trypsin, and chymotrypsin were purchased from Sigma Chemical Co. Staphylococcus aureus V8 was purchased from Boehringer Mannehim (Indianapolis, IN). Sodium sulfite and 5,5'-dithiobis-2-nitrobenzoic acid (Ellman's reagent) were purchased from Aldrich Chemical Co. (Milwaukee, WI). HPLC-grade acetonitrile was purchased from Fisher Scientific (Fair Lawn, NJ) and HPLC-grade water was obtained from a Barnstead Nanopure ultrapure water system (DuBuque, IA).

\section{Enzymatic Digestion}

RNase B was denatured by S-sulfonation according to the method of Thannhauser et al. [32] prior to digestion with trypsin. The sulfonation reagent, 2-nitro-5thiosulfobenzoate (NTSB), was synthesized according to the following procedure [33]: Ellman's reagent (100 $\mathrm{mg}$ ) was dissolved in $10 \mathrm{~mL}$ of $1 \mathrm{M} \mathrm{Na}_{2} \mathrm{SO}_{3}$, the $\mathrm{pH}$ adjusted to 7.5, and oxygen bubbled through the solution until it turned yellow (final solution $=50 \mathrm{mM}$ NTSB $/ 1 \mathrm{M} \mathrm{Na}_{2} \mathrm{SO}_{3}$ ). RNase B (1 mg) was dissolved in $50 \mu \mathrm{L}$ of $400 \mathrm{mM}$ tris buffer $(\mathrm{pH} 8.2)$ and $150 \mu \mathrm{L}$ of 8 $\mathrm{M}$ guanidine $\mathrm{HCl}$, then $\mathrm{Na}_{2} \mathrm{SO}_{3}(5 \mathrm{mg})$ was added and the mixture incubated at $37^{\circ} \mathrm{C}$. After $30 \mathrm{~min}, 100 \mu \mathrm{L}$ of the NTSB solution was added (deep yellow color), and the mixture incubated at $37^{\circ} \mathrm{C}$ for another $30 \mathrm{~min}$. The sulfonation was quenched by lowering the $\mathrm{pH}$ to 3 with a few drops of $96 \%$ formic acid and the reaction mixture desalted by elution through a PD-10 column from Pharmacia (Uppsala, Sweden) with $3.5 \mathrm{~mL}$ of 50 $\mathrm{mM} \mathrm{NH} \mathrm{NH}_{4} \mathrm{HCO}_{3}$. S-sulfonated $\left(\mathrm{S}_{-} \mathrm{SO}_{3}\right)$ RNase $\mathrm{B}$ was digested with trypsin for $12 \mathrm{~h}$ at $37^{\circ} \mathrm{C}$ at $\mathrm{pH} 8$ with a substrate-to-enzyme ratio of 50:1 $(\mathrm{w} / \mathrm{w})$.

Ovomucoid was reduced and carboxymethylated [34] prior to digestion with trypsin and chymotrypsin. Typically, the protein (1-2 mg) was dissolved in $50 \mu \mathrm{L}$ of $100 \mathrm{mM}$ tris buffer $(\mathrm{pH} \mathrm{8.2)}$ and $150 \mu \mathrm{L}$ of $8 \mathrm{M}$ guanidine $\mathrm{HCl}$, the $\mathrm{pH}$ adjusted to $8-8.5$ with a few drops of $1 \mathrm{M} \mathrm{NH}_{4} \mathrm{OH}$ and DTT $(5 \mathrm{mg} / 100 \mu \mathrm{L} 1 \mathrm{M}$ $\mathrm{NH}_{4} \mathrm{OH}$ ) added in at least a 40 -fold molar excess of each disulfide bond present. The mixture was incubated at $37^{\circ} \mathrm{C}$ for $2 \mathrm{~h}$ and iodoacetic acid $(19 \mathrm{mg} / 100$ $\mu \mathrm{L} 1 \mathrm{M} \mathrm{NH}_{4} \mathrm{OH}$ ) in a 2-3-fold molar excess of DTT was added in the dark. The $\mathrm{pH}$ was readjusted to 8-8.5 with $1 \mathrm{M} \mathrm{NH}_{4} \mathrm{OH}$ and monitored at intervals for $30 \mathrm{~min}$. The reaction mixture was desalted with a PD-10 column as above. Reduced and carboxymethylated (RCM) ovomucoid was treated with trypsin and then chymotrypsin for $12 \mathrm{~h}$ each at $37^{\circ} \mathrm{C}$ at $\mathrm{pH} 8$ with a substrate-to-enzyme ratio of 50:1 (w/w). ASF was reduced, carboxymethylated, and treated with trypsin and chymotrypsin as described above with a substrateto-enzyme ratio of $50: 1(\mathrm{w} / \mathrm{w})$; a portion was subsequently treated with Staphyloccus aureus protease V8 with a substrate-to-enzyme ratio of 25:1 ( $w / w)$. Fetuin was reduced and carboxymethylated as described above and treated with trypsin as above with a substrate-to-enzyme ratio of $50: 1$. Typically, $10 \mathrm{mg} / \mathrm{mL}$ of 
glycoprotein was reduced, carboxymethylated, and digested with proteases such that $20 \mu \mathrm{L}$ injections in the LC/MS system contained peptides from 1-1U nmol of total protein.

\section{Solid-Phase Extraction}

Peptides and glycopeptides from the proteolytic digests were fractionated by solid-phase extraction (SPE) cartridges $\left(C_{18}, 40-\mu \mathrm{m}\right.$ particle size) from Baker (Phillipsburg, NJ). A few drops of $1 \mathrm{M}$ TFA were added to lower the $\mathrm{pH}$ to 2 , and the mixture loaded onto a $3-\mathrm{mL}$ SPE cartridge (200-mg packing) that had been equilibrated with $3 \mathrm{~mL}$ each of methanol, acetonitrile, and $0.1 \%$ TFA. The column was washed sequentially with $3 \mathrm{~mL}$ each of $0.1 \%$ TFA, $10 / 90 \mathrm{CH}_{3} \mathrm{CN} / \mathrm{H}_{2} \mathrm{O}(0.1 \%$ TFA), $80 / 20 \quad \mathrm{CH}_{3} \mathrm{CN} / \mathrm{H}_{2} \mathrm{O} \quad(0.1 \%$ TFA $), \quad 30 / 70$ $\mathrm{CH}_{3} \mathrm{CN} / \mathrm{H}_{2} \mathrm{O}\left(0.1 \%\right.$ TFA), and $50 / 50 \mathrm{CH}_{3} \mathrm{CN} / \mathrm{H}_{2} \mathrm{O}$ ( $0.1 \%$ TFA). The collected fractions were concentrated with a Savant (Farmingdale, NY) Speed-Vac and reconstituted in $100-200 \mu \mathrm{L} 50 / 50 \mathrm{CH}_{3} \mathrm{CN} / \mathrm{H}_{2} \mathrm{O}(0.5 \%$ $\mathrm{HCOOH}$ ) or mass spectral analysis by ion spray LC/MS or continuous infusion MS/MS.

\section{Microbore Liquid Chromatography}

The proteolytic digests of RCM ovomucoid, RCM asialofetuin, and RCM fetuin were separated with a YMC (Morris Plains, NJ) Basic (C8) $1 \mathrm{~mm}$ i.d. $\times 150$ $\mathrm{mm}$ column. Typically, a gradient from $\mathrm{H}_{2} \mathrm{O}$ to $50 / 50$ $\mathrm{CH}_{3} \mathrm{CN} / \mathrm{H}_{2} \mathrm{O}$ in $0.05 \%$ TFA was performed in $50 \mathrm{~min}$ at $40 \mu \mathrm{L} / \mathrm{min}$. Samples containing 1-10 $\mathrm{nmol}(20 \mu \mathrm{L})$ were injected directly from buffered digest with a Rheodyne (Cotati, CA) model 9125 injector equipped with a 20- $\mu \mathrm{L}$ sample loop. An Applied Biosystems Inc. (ABI, Foster City, CA) model 140-A dual-syringe micro LC pump was used with a $52-\mu \mathrm{L}$ static mixer instead of the dynamic mixer supplied with the original instrument. Peptides were detected at $214 \mathrm{~nm}$ with an ABI model 757 absorbance detector equipped with a 1.2- $\mu \mathrm{L}$ flow cell. The entire flow was directed to an ion spray interface with a minimum of connecting tubing $\left(0.005^{\prime \prime}\right.$ i.d. PEEK, Oak Harbor, WA) between detectors.

\section{Mass Spectrometry}

A Sciex (Thornhill, Ontario) TAGA 6000E atmospheric pressure ionization (API) tandem triple quadrupole mass spectrometer updated to an API-III system with a $m / z$ 10-2400 mass range and a Macintosh II data acquisition system was used. Samples were introduced into the mass spectrometer continuously at 2-4 $\mu \mathbf{L} /$ min by infusion pump (Harvard Apparatus, South Natick, MA) for CID MS/MS experiments. The inhouse constructed ion spray interface was maintained at 3-4 kV and positioned at a $45^{\circ}$ angle approximately $1 \mathrm{~cm}$ away from the ion-sampling orifice. A coaxial nitrogen flow was maintained at $60-65$ psi as nebulizing gas. The conical skimmer orifice $(100-120 \mu \mathrm{m}$ i.d.) was bathed in an ultrapure nitrogen curtain gas that was countercurrent to the flow of the sprayer and served to keep small particles and droplets from entering the analyzer region of the mass spectrometer. The mass axis of each mass resolving quadrupole $\left(Q_{1}\right.$ and $Q_{3}$ ) was calibrated with the ammonium adducts of polypropylene glycol (PPG) by continuous infusion (4 $\mu \mathrm{L} / \mathrm{min})$ of a solution of PPG $1000(0.1 \mathrm{mM})$ and PPG $2000(0.2 \mathrm{mM})$ in $80 / 20 \quad \mathrm{CH}_{3} \mathrm{CN} / \mathrm{H}_{2} \mathrm{O}(3 \mathrm{mM}$ $\left.\mathrm{NH}_{4} \mathrm{OAc}\right)$. Each mass-resolving quadrupole $\left(\mathrm{Q}_{1}, \mathrm{Q}_{3}\right)$ was operated at a resolution of at least $20-50 \%$ valley between peaks one mass unit apart for $m / z$ 10-2000 and $50-70 \%$ valley for $m / z 2000-2400$ for mass calibration. A declustering potential of $30 \mathrm{~V}$ was maintained by floating the orifice at $60 \mathrm{~V}$ and the first RF-only quadrupole $\left(Q_{0}\right)$ at $30 \mathrm{~V}$.

For CID MS/MS studies the resolution of $Q_{1}$ was lowered to transmit a mass window centered on the precursor mass-to-charge ratio that was $2-3 \mathrm{u}$ wide at half height; the second mass-resolving quadrupole $\left(Q_{3}\right)$ was operated at a resolution that transmitted ions that were $1 \mathrm{u}$ wide at half height. The collision cell $\left(Q_{2}\right)$ was offset at 40-50 V lower than $Q_{0}$ such that doubly charged tryptic glycopeptides were accelerated to a collision energy of $80-100 \mathrm{eV}$ laboratory frame. The collision gas (Ar) thickness was set to $2.3-2.6 \times 10^{14}$ atoms $/ \mathrm{cm}^{2}$ such that the pressure in the analyzer was increased from $2.1 \times 10^{-5}$ torr to $2.5-3.0 \times 10^{-5}$ torr. Precursor ions were collisionally preheated in the declustering region between the orifice and the first $R \Gamma$-only quadrupole $\left(Q_{0}\right)$ by maintaining a declustering potential of $65-70 \mathrm{~V}$. For the doubly charged glycopeptide of RNase $B$ a mass range from $m / z 10$ up to twice the precursor mass-to-charge ratio was scanned by peak hopping every mass unit with a dwell time of $5 \mathrm{msec}$ at each step; multichannel averaging of 32 scans was used to increase the signal-tonoise ratio. For the doubly charged glycopeptides of ovomucoid a mass range $m / z$ 160-2400 was scanned as above with a dwell time of 5 or $10 \mathrm{msec}^{\prime} 64$ scans were averaged. Raw data were smoothed once with software available from Sciex.

For LC/CID/MS experiments fragmentaion of glycopeptides was achieved in the free-jet expansion region between the orifice and the first RF-only quadrupole $\left(Q_{0}\right)$ by CID with the nitrogen curtain gas [31]. The orifice was maintained at $90-130 \mathrm{~V}$ and $Q_{0}$ was maintained at $30 \mathrm{~V}$ to produce declustering potentials of $60-100 \mathrm{~V}$, respectively. The first mass-resolving quadrupole $\left(Q_{1}\right)$ was operated at unit resolution (20-50\% valley from $m / z 10$ to $2000,50-70 \%$ valley from $m / z 2000$ to 2400 ) and scanned by peak-hopping each mass unit from $m / z 200$ to 2400 every 6 sec.

\section{Results and Discussion}

\section{S-Sulfonation of Glycoproteins}

S-Sulfonation of disulfide bonds is a convenient, quantitative method for the denaturation of folded proteins prior to enzymatic hydrolysis that is not sensitive to the oxidative or photolytic side reactions [32] that occur with the reduction and carboxymethylation pro- 
cedure. The S-sulfonated proteins are excellent substrates for proteolytic hydrolyses [32], although solubility sometimes is a problem. HPLC mapping of the peptides obtained by tryptic digestion of S-sulfonated RNase A has been reported previously [33]; therefore, this procedure was chosen for the glycosylated protein, RNase B. An added advantage to the S-sulfonation procedure with electrospray is the observation that molecules with sulfonate and sulfate groups exhibited very good sensitivity with ion spray in the negative ion mode [35, 36]. This phenomenon was not investigated fully for electrospray of proteins and remains an area for future work.

\section{Continuous Infusion of Glycopeptide Fractions for CID MS/MS}

The heterogeneity inherent to the extensive glycosylation of proteins creates an analytical challenge with respect to sensitivity for the determination of the glycoforms. Upon specific proteolysis of a glycoproteir, glycopeptides are divided into subpopulations that each contain the same peptide portion but differ in the attached carbohydrate. This places a great demand on the sensitivity of a technique such as MS/MS that fragments the individual glycopeptides and further divides the ion current into many new signals. One solution to this problem is to lower the resolution of the mass-resulving quadrupoles in order to focus more ions with wider peak widths to give greater intensity. With a triple quadrupole mass spectrometer this has been reported by detuning the first mass-resolving quadrupole $\left(\mathrm{Q}_{1}\right)$ to obtain as much precursor ion intensity as possible [37]. However, if the resolution of the first mass-resolving quadrupole $\left(Q_{1}\right)$ is lowered too much, it may pass more than just the desired precursor ion, which may result in a mixed CID mass spectrum. If the resolution of the second mass resolving quadrupole $\left(\mathrm{Q}_{3}\right)$ is also lowered, peaks that differ by one mass unit in the resulting product ion spectrum may become indistinguishable.

A compromise is achieved by establishing moderately lowered mass resolution in $Q_{1}$ while maintaining unit mass resolution in $Q_{3}$ [37] and averaging the signal acquired over multiple scans. Any interfering ions resulting from the detuning of $Q_{1}$ that would produce a mixed mass spectrum would be readily apparent in a unit-resolved CID mass spectrum. For the purpose of obtaining representative CID mass spectra of the glycopeptides studied in this work, LC/MS/MS analysis of glycopeptide mixtures directly from the enzymatic digest of the glycoprotein was found to be impractical. CID MS/MS spectra of the glycopeptides containing more structurally significant ions were obtained by prior fractionation of the peptide mixture with solid-phase extraction techniques and continuous infusion of the partially purified mixture. Multichannel averaging was used to obtain a sufficient signal-to-noise ratio so the resolution of the second mass resolving quadrupole $\left(Q_{3}\right)$ was not low- ered and therefore the quality of the data was not compromised.

\section{Collision-Induced Dissociation Mass Spectra of Glycopeptides}

High-Mannose Type: RNase B. The CID MS/MS experiments discussed below were all performed by continuous infusion of partially purified proteolytic digest mixtures of glycoproteins (see Experimental section for details). Doubly charged tryptic peptides have desirable properties as precursor ions for CID MS/MS, because abundant fragmentation is observed with relatively low collision energies in a triple quadrupole $(<100 \mathrm{eV})$ to produce a nearly complete C-terminal (" $Y$ ') ion series of sequence information [26]. Multiply charged tryptic peptides are commonly observed because of the presence of either a lysine or arginine at the C-terminus. Doubly charged ions result from protonation of the basic residues of these amino acids in addition to the basic amino terminus, and triply charged ions may result if a histidine is present [38].

Multiply charged precursor ions yield increased fragmentation in CID experients because of increased acceleration and internal charge repulsion, both proportional to the charge slale [39]. Doubly charged ions are logical choices for MS/MS because the resulting product ions can contain only doubly and singly charged ions, the latter being more commonly observed. Focusing a precursor ion with a charge state higher than two for CID MS/MS produces a product ion set that has ambiguous charge state information, because each product ion can be singly or multiply charged. The charge state information in the isotopic cluster of each product ion is often unresolvable in MS/MS experiments with a triple quadrupole mass spectrometer because of the increased energy spread of the product ions. Although charge states higher than two were observed for the glycopeptides investigated in this work, only the doubly charged precursor ions were chosen for CID MS/MS of the tryptic glycopeptides for the reasons discussed above.

The full-scan CID MS/MS spectrum of the highmannose tryptic glycopeptide from $\mathrm{S}_{-} \mathrm{SO}_{3}$ RNase $\mathrm{B}$ is shown in Figure 1 (structure shown in inset). As expected, the doubly charged precursor ion yielded abundant fragmentation at the collision energy used (80-100 eV). Several structural features are apparent in this mass spectrum. At the low mass-to-charge region the characteristic oxonium ions are observed arising from cleavage at the glycosidic linkage with charge retention on the sugar: $\mathrm{Man}^{+}\left(m / z\right.$ 163), GlcNAc${ }^{+}$ $\left(m / z\right.$ 204), ManMan $^{+}\left(m / z\right.$ 325), and ManGlcNAc ${ }^{+}$ $(m / z 366)$. The relative intensities of the $\mathrm{Man}^{+}$and $\mathrm{ManMan}^{+}$oxonium ions are greater than those of the more stable GlcNAc-containing oxonium ions, because the proximal mannoses can be lost from the nonreducing terminus by only one bond cleavage. The GlcNAccontaining fragments must arise by at least two bond cleavages from the chitobiose core of the glycopeptide. 


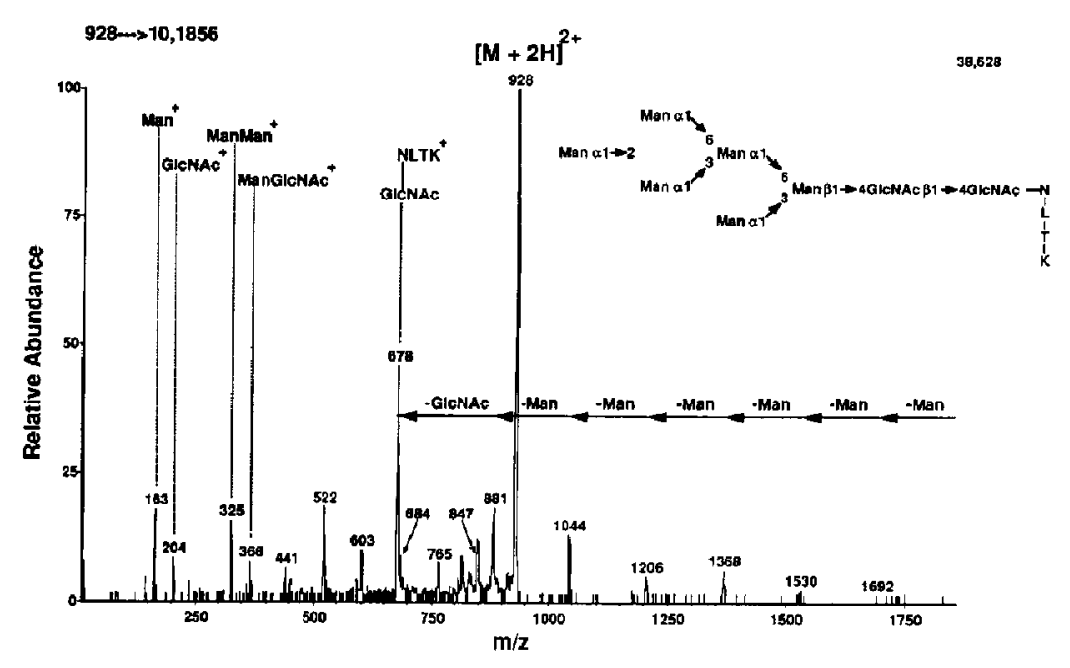

Figure 1. Full-scan CID MS/MS spectrum of the doubly charged precursor ion at $m / z 928$ for the glycopeptide from RNase $B$ with the structure shown in the inset. Collision energy for the doubly charged precursor was $80 \mathrm{eV}$. Data were averaged for 32 scans.

At the high mass-to-charge region is a complete series of Y-type ions corresponding to consecutive losses of monosaccharide residues with hydrogen rearrange ment and charge retention on the reducing (peptide) end of the glycopeptide [11].

The intensities of the individual ions do not reflect the branching pattern of the oligosaccharide portion of the glycopeptide as has been reported with high-energy CID of ABEE derivatives of oligosaccharides [13]. The multiple collisions at low energy that occur in a triple quadrupole probably cause multiple bond cleavages, and single bond cleavages are necessary to preserve branching information. Huwever, partial sequence information can be available. For example, the full-scan CID MS/MS spectrum of the doubly charged precursor ion at $m / z 928$ for the glycopeptide from RNase B has been shown in Figure 1. The loss of 162 mass units from each of the high-mass ions in the series is characteristic of Hex residues and reflects the high-mannose nature of the attached oligosaccharide. The difference of one more mass unit difference (163u) between the ions at $m / z 881$ and 1044 is due to the peak-hopping acquisition mode of the mass spectrometer used in this work, which assigns ions to the nearest integer mass. The fragment species (GlcNAc) ${ }_{2}-$ N-L-T-K ( $m / z$ 881.4, monoisotopic mass) was detected at $m / z 881$ and the fragment species $\operatorname{Man}\left(\mathrm{GlcNAc}_{2}\right)$ N-L-T-K ( $m / z$ 1043.5, monoisotopic mass) was detected at $m / z$ 1044. A complementary doubly charged ion series with charge retention on the tryptic peptide is observed at $m / z 441,522,603,684,765$, and 847 , each spaced by $81 \mathrm{u}$ except for the difference of $82 \mathrm{u}$ between $m / z \quad 765$ and 847 . The fragment species $\operatorname{Man}_{4}\left(\mathrm{GlcNAC}_{2}\right)-\mathrm{N}-\mathrm{L}-\mathrm{T}-\mathrm{K}\left(\mathrm{m} / \mathrm{z} 765.4,[\mathrm{M}+2 \mathrm{H}]^{2+}\right.$ monoisotopic mass) was detected at $m / z 765$ and the fragment species $\mathrm{Man}_{5}\left(\mathrm{GlcNAc}_{2}\right)-\mathrm{N}-\mathrm{L}-\mathrm{T}-\mathrm{K}(\mathrm{m} / \mathrm{z} 846.4$, $[\mathrm{M}+2 \mathrm{H}]^{2+}$ monoisotopic mass; $m / z$ 846.9, $[\mathrm{M}+$ $2 \mathrm{H}]^{2+}$ average mass) was detected at $m / z$ 847. The observed mass of peptides shifts from the monoisotopic mass toward the average mass as the mass increases [40]. The assignment of a very weak ion at $m / z$
1692 is strengthened by the presence of the doubly charged ion at $m / z 847$.

An interesting feature of the mass spectrum shown in Figure 1 is the abundance of ions representative of $O$-glycosidic cleavage to yield both reducing- and nonreducing-terminal fragments without any evidence of amide bond clcavage of the peptide backbone. The collision energy used was sufficient to cause abundant fragmentation of other doubly charged tryptic peptides to yield some sequence information [26]. For a constant collision energy, cleavage of the glycosidic bonds of an oligosaccharide appears more facile than rupture of the secundary amide bond of a peplide for biopolymers of comparable mass [41]. When both types of bonds are present in a glycopeptide, the peptide backbone appears to remain intact, while all O-glycosicide bonds are cleaved. It may be that low-energy CID is a selective process such that weaker bonds can absorb the energy of a collision and protect stronger bonds from cleavage. In fact, the $N$-glycosidic linkage between the core GICNAC and Asn residue is apparently stronger than the corresponding $\mathcal{U}$-glycosidic bonds, because the ion at $m / z 678$ is reasonably intense. The molecular weight of the peptide can be determined from this ion by subtracting the mass of a GlcNAc residue (203 u).

The lack of peptide backbone cleavage in the CID MS/MS spectra of glycopeptides is a fortuitous result, because the peptide can serve as both an ionizable, charge-retaining and fragmentation-directing group that does not further complicate the mass spectrum except for the addition of mass. This could be a convenient "derivative" for oligosaccharides that naturally occur attached to peptides such as those found in glycoproteins. The remaining examples are shown for the complex tryptic glycopeptides derived from two of the four $\mathrm{N}$-linked sites of ovomucoid.

Complex-Type: Ovomucoid Glycopeptides. The N-linked oligosaccharides of ovomucoid occur at the residues ${ }^{10} \mathrm{Asn},{ }^{53} \mathrm{Asn},{ }^{69} \mathrm{Asn}$, and ${ }^{75} \mathrm{Asn}$ of the amino acid se- 


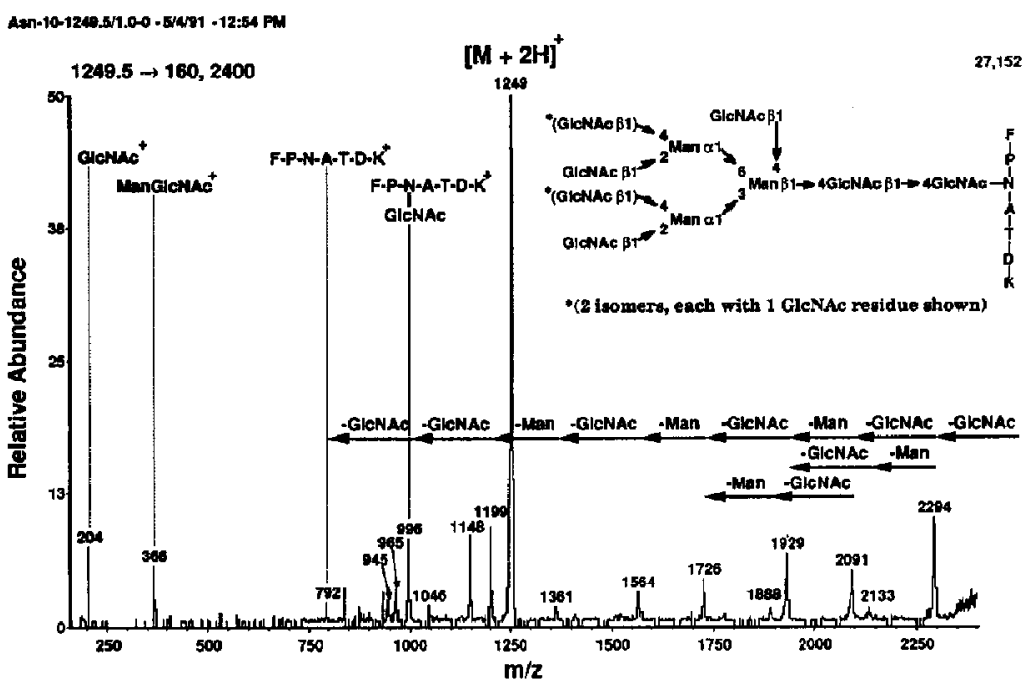

Figure 2. Full-scan CID MS/MS spectrum of the doubly charged precursor ion at $\mathrm{m} / \mathrm{z}$ 1249.5 for the glycopeptide from ovomucoid with the structure shown in the inset. Collision energy for the doubly charged precursor was $80 \mathrm{eV}$. Data were averaged for 64 scans. quence [42, 43]. Digestion of the reduced and carboxymethylated glycoprotein with trypsin and chymotrypsin yields small trypticlike glycopeptides containing ${ }^{10} \mathrm{Asn},{ }^{53} \mathrm{Asn}$, and ${ }^{75} \mathrm{Asn}[43]\left({ }^{69} \mathrm{Asn}\right.$ is contained in a nontrypticlike glycopeptide) that desorb as doubly charged ions for subsequent CID MS /MS. Further digestion with chymotrypsin (or chymotrypticlike activity with trypsin) results in cleavage at ${ }^{50} \mathrm{Phe}$ to produce the trypticlike glycopeptide G-T-N-I-S-K containing ${ }^{53} \mathrm{Asn}$. The novel complex-type sugar chains occurring in ovomucoid have been well characterized $[44,45]$. The common structure is a chitobiose-trimannosyl core containing up to $6 \beta$-linked GlcNAc residues attached to the 2-, 4-, and 6-position of the $\alpha$-linked mannoses and the 4-position of the $\beta$-linked mannose [44]. Minor structures contain one or two $\beta$-linked galactose residues to the proximal GlcNAc thereby creating $N$-acetyllactosamine units, and various combinations of attachment points of the GlcNAc residues to the chitobiose-trimannosyl core [45].

The ovomucoid glycopeptides were fractionated with SPE cartridges similar to the procedure used to purify the RNase B glycopeptides and the collected fractions were screened by continuous infusion for predicted [43] precursor mass-to-charge values. The CID MS /MS spectrum of a triantennary complex tryptic glycopeptide at ${ }^{10}$ Asn of ovomucoid is shown in Figure 2. The structure of the glycopeptide (inset in Figure 2) shows 4 GlcNAc residues attached $\beta 1 \rightarrow 4$ to the $\beta$-linked mannose, $\beta 1 \rightarrow 2$ to the two $\alpha$-linked mannoses, and $\beta 1 \rightarrow 4$ to either one or the other $\alpha$-linked mannose. This structure accounts for $21 \%$ of the total glycan occurring at this glycosylation site, as measured by FAB/MS with ABEE derivatives [43].

The mass spectrum shown in Figure 2 is similar to that obtained with the tryptic glycopeptide derived from RNase B (see Figure 1). Oxonium ions corresponding to GicNAc${ }^{+}$and ManGlcNAc ${ }^{+}(m / z 204$ and 366 , respectively) were observed preferentially, the former ion presumably due to stabilization of the positive charge by the acetamido group of the GlcNAc residue [46]. Additionally, only one bond must be broken to generate these nonreducing terminal ions. A Y-type ion series was present, which shows the consecutive losses of the monosaccharide residues with hydrogen rearrangement and charge retention on the peptide [11] at $m / z 2294,2133,2091,1929,1888,1726$, $1564,1361,1199,996$, and 792 . The spacing between each ion corresponds to a loss of either mannose (162 u) or GICNAc (203 u) residue with the exception of the weak ion at $m / z 2133$ and the ion corresponding to the peptide at $m / z$ 792. The discrepancy with the former ion is due to uncertainty with mass assignment \pm 1 w with peaks of low signal-to-noise ratios. The difference of $204 \mathrm{u}$ between the ions at $m / z 792$ and 996 is due to the mass defect round up discussed previously. The peak widths of product ions in this mass spectrum increase with mass-to-charge ratio and reflect the mass-dependent kinelic energy of product ions analyzed with a constant offset between the collision cell and second mass-resolving quadrupole [47, 48]. A doubly charged ion series (separated by $81 \mathrm{u}$ or $101.5 u$ ) occurs at $m / z 1148,1046,965$, and 945 , which is complementary to the major ion series observed at high mass. The oligosaccharide composition and partial sequence in Hex and HexNAc units can be determined with the complete series of reducing-terminal ions. By following the loss of sugar units through the $\beta$-mannosylchitobiose core (ions at $m / z 1199,996,792$ ) the mass of the original peptide may be determined. The presence of tragments at $m / z 996$ in Figure 2 and $m / z 678$ in Figure 1 is reminiscent of the information available from endo-H-like cleavages previously reported [49].

The CID MS/MS spectrum of a related tetraantennary complex trypticlike glycopeptide at ${ }^{53} \mathrm{Asn}$ of ovomucoid displayed many similarities to the mass spectra described in the preceding examples. The chy- 
motryptic site was on the N-terminal side of the glycosylation site, so the doubly charged nature of the glycopeptide was conserved. The same oxonium ions $(m / z 204,366)$ were observed at low mass, and a complete singly charged series of reducing terminus ions with a partial series of doubly charged ions suggests the possible sugar composition and partial sequence of the oligosaccharide.

\section{Screening for Glycopeptides in Proteolytic Digest Mixtures of Glycoproteins by LC/CID/MS}

The facile production of sugar oxonium ions from glycosylated peptide precursors generated in the collision cell $\left(Q_{2}\right)$ by CID MS/MS experiments suggests a strategy for the selective detection of glycopeptides in enzymatic digest mixture of glycoproteins. Fragment ions containing $N$-acetylated hexosamine are likely candidates for glycopeptide screening, because the nitrogen atom at position 2 stabilizes the positive charge [46] and oxonium ions at $m / z 204$ (HexNAc ${ }^{+}$) and $m / z 366$ (HexHexNAc ${ }^{+}$) have been observed in CID MS/MS spectra of glycopeptides. Although these oxonium ions can be liberated readily by a single bond cleavage from nonreducing terminal GlcNAc or $N$ acetyllactosamine units, respectively, these ions may be produced by multiple bond cleavages from nonterminal sugar units (for example, the CID MS/MS spectrum of RNase B high-mannose glycopeptides shown in Figure 1). There is also the possibility that the ion selected may derive from sources other than carbohyArate. LC/MS/MS precursor ion scanning for oxonium ions has been exploited by others for the purpose of glycopeptide screening [50].

An alternative approach to glycopeptide screening could be the production of the same oxonium ions observed by MS/MS. The fragmentation of glycopeptides in the declustering region of the API mass spectrometer [31] lacks the pre-mass selection (and mixture analysis capability) of true MS/MS, but produces comparable fragmentation information with better sensitivity. We call this collision-induced dissociation in the declustering region of an API mass spectrometer "up front" CID, and suggest this is a practical means for obtaining reproducible structural information through fragmentation of drugs [51], peptides [52, 53], and proteins $[53,54,55]$. An orifice potential of $90 \mathrm{~V}$ was chosen to maximize the intensities of the ion signals in the range $m / z$ 800-1600 for LC/MS (and energetically preheat the ions for subsequent MS/MS); however, the increased declustering potential of $60 \mathrm{~V}$ was found to also produce oxonium ions from glycopeptides by nonreducing terminal charge retention (see below). LC/MS experiments could be designed to defect the characteristic oxonium ions produced in the declustering region without resorting to MS/MS. By utilizing only a single stage of mass filtering the problems associated with LC/MS/MS regarding reduced sensitivity from poor ion transmission and scattering losses may possibly be avoided. This LC/CID/MS approach could be used to screen for glycopeptides by scanning a mass range sufficient to detect the oxonium ions along with the precursor glycopeptides.

A $20-\mu \mathrm{L}$ injection of a proteolytic digest mixture resulting from treatment of RCM ovomucoid (10 $\mathrm{mg} / \mathrm{mL}$ ) with trypsin and chymotrypsin was analyzed by LC/MS. The extracted ion chromatograms for $m / z$ 204 and 366 , and the total ion chromatogram from the full-scan LC/MS experiment scanned over $m / z$ 200-2400 with a declustering potential of $60 \mathrm{~V}$ revealed where the diagnostically useful $m / z 204$ and 366 oxonium ions were located in the LC/CID/MS total ion current profile (not shown here). The use of a moderately high declustering potential increases the abundance of oxonium ions and reduces the abundance of solvent chemical noise in the lower mass region [57]. Chymotrypsin used in this case produced a nonspecific cleavage [56] at ${ }^{68} \mathrm{Met}$ such that the glycopeptide produced by chymotryptic activity of trypsin (E-T-V-P-M-N-C-S-S-Y) [43] was further digested to a smaller peptide (N-C-S-S-Y). The location of glycopeptides in the full-scan data set is facilitated by comparing the extracted ion chromatograms corresponding to the oxonium ions of $m / z 204$ and 366. The concurrent rise and fall of both ion current chromatograms suggests the possibility that a glycopeptide is present at a particular retention time, and background subtraction of the scans composing that chromatographic peak can reveal an interpretable full-scan $\mathrm{LC} / \mathrm{CID} / \mathrm{MS}$ spectrum that can facilitate determining whether this is the case.

Figure 3 shows full-scan mass spectra obtained from the $m / z 204$ and 366 extracted ion current chromatographic profiles observed in the proteolytic digest mixture described above resulting from treatment of RCM ovomucoid (10 $\mathrm{mg} / \mathrm{mL})$ with trypsin and chymotrypsin where LC/CID/MS analysis is provided for the two glycopeptides corresponding to the $\mathrm{N}$-linked glycosylation sites of ovomucoid at ${ }^{10} \mathrm{Asn}$ and ${ }^{53} \mathrm{Asn}$, respectively. The doubly charged ions (evidenced by the spacing of $203 / 2 \mathrm{u}$ ) at $m / z 843,945,1047,1148$, 1250, and 1352 of Figure 3a correspond to the species containing 0-5 GlcNAc residues, respectivley, attached to the trimannosyl chitobiose core at ${ }^{10} \mathrm{Asn}$ of the peptide F-P-N-A-T-D-K [43]. Also present at low mass are the oxonium ions at $m / z 204$ and 366 that were used to locate these particular mass spectra in the full-scan data set. The mass spectrum of a different glycopeptide containing the peptide G-T-N-I-S-K and the same oligosaccharides as the previous example is shown in Figure 3b. Along with the oxonium ions at $m / z 204$ and 366 is the doubly charged series of ions corresponding to the glycoforms containing 0-6 GlcNAc residues at $m / z 757,858,960,1061,1163,1265$, and 1367 attached to the trimannosyl chitobiose core at ${ }^{53} \mathrm{Asn}$ of ovomucoid. As seen in the preceding case the species corresponding to the peptide and trimannosyl chitobiose core sugar $(\mathrm{m} / z 757)$ is probably produced in the declustering region of the mass spectrometer and is not normally found in native ovomucoid [43]. 

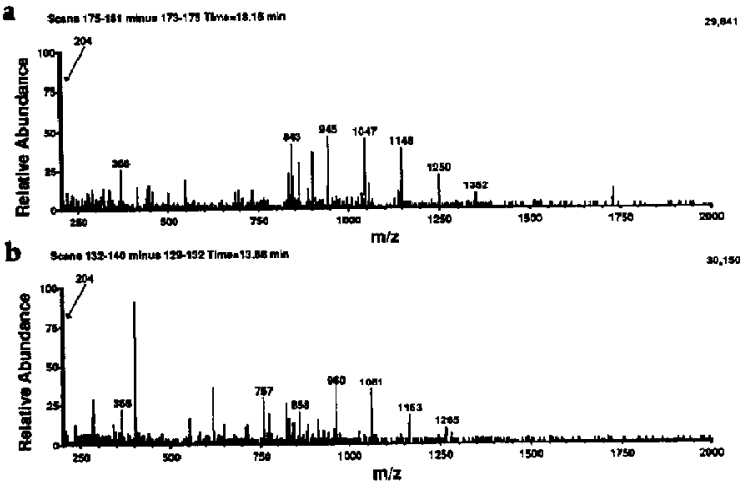

Figure 3. (a) Background-subtracted, averaged mass spectrum corresponding to the glycopeptides contairing ${ }^{10}$ Asn of ovomucoid from a chromatographic peak from the LC/CID/MS total ion chromatogram obtained from the analysis of $\mathrm{RCM}$ ovomucoid digested with trypsin and chymotrypsin (ca. $10 \mathrm{nmol}$ injected). (b) Background-subtracted, averaged mass spectrum corresponding to the glycopeptides containing ${ }^{53} \mathrm{Asn}$ of ovomucoid from the chromatographic peak of the LC/CID/MS total ion chromatogram obtained from the analysis of RCM ovomucoid digested with trypsin and chymotrypsin (ca. $10 \mathrm{nmol}$ injected).

A significant limitation of the LC/CID/MS approach for detecting glycopeptides is that the relative intensities of the ions corresponding to the individual glycoforms do not accurately reflect the actual distribution of the glycoforms. For example, the relative intensities of the ions corresponding to the glycoforms shown in Figure 3 reflect the collision-induced fragmentation processes that occur in the declustering region. The lower glycoforms are produced from the higher glycoforms by sequential loss of GlcNAc residues, some of which appear as oxonium ions. Another limitation is that ions appear in the LC/CID/MS spectra that may not originate from the glycopeptide, but from chemical background or coeluting compo-

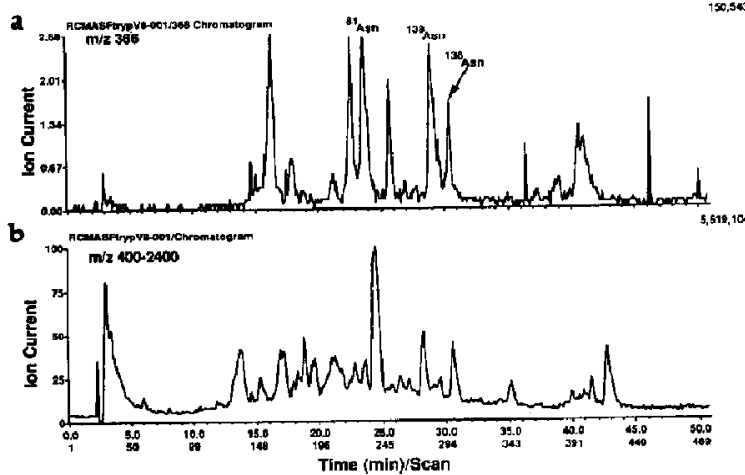

Figure 4. (a) LC/CID/MS extracted ion chromatogram for $m / z$ 366 from a $20-\mu \mathrm{L}$ injection of RCM ASF digested with trypsin and Staph. aureus V8 (ca. $10 \mathrm{nmol}$ injected). Data were acquired by scanning $Q_{1}$ from $m / z 300$ to 2400 in $1.0 \mathrm{u}$ steps for 2.86 msec/step (6.2 sec/scan) with a declustering potential of $60 \mathrm{~V}$. Glycopeptides are tentatively identified as shown. Other peaks are unidentified. (b) Full-scan tatal ion chromatogram replotted from $m / z 400$ to 2400 . See text for chromatographic conditions. nents (the unlabeled peak at $m / z 403$ of Figure 3b).

Another example of the LC/CID/MS approach for glycopeptide screening is demonstrated for the glycoprotein asialofetuin (ASF). Fetuin contains $3 \mathrm{~N}$-linked glycosylation sites (at ${ }^{81} \mathrm{Asn},{ }^{138} \mathrm{Asn}$, and ${ }^{158} \mathrm{Asn}$ ) and at least $3 \mathrm{O}$-linked glycosylation sites [58]. The structures of both the $\mathrm{O}$-linked $[59,60]$ and $\mathrm{N}$-linked oligosaccharides attached to fetuin have been extensively studied $[61,62,63,64,65]$. The $\mathrm{N}$-linked glycosylation sites cannot be isolated into convenient small glycopeptides by digestion with trypsin alone; therefore, a subsequent digestion with Staph. aureus V8 was performed. The LC /CID/MS extracted ion chromatogram for $m / z$ 366 (Figure 4a) suggests the possible chromatographic locations of the glycopeptides that would otherwise be indistinguishable in the full-scan ion chromatogram (Figure 4b). The HexHexNAc oxonium ions at $\mathrm{m} / z$ 366 shown in Figure 4a were produced in $Q_{0}$ with a declustering potential of $60 \mathrm{~V}$. Because of the complexity of this glycoprotein only two of the $\mathrm{N}$-linked sites (from three of the chromatographic peaks in Figure 4a) were tentatively identified by comparing the mass spectra to the expected glycopeptides [61]. The glycopeptide corresponding to ${ }^{81}$ Asn that eluted at 23.7 min shown in Figure 4a contains amino acid residues 69-85 resulting from V8 cleavage at ${ }^{68} \mathrm{Glu}$ and tryptic cleavage at ${ }^{85} \mathrm{Arg}$. The glycopeptides corresponding to ${ }^{138} \mathrm{Asn}$ result from tryptic cleavage at ${ }^{141} \mathrm{Arg}$; the major species that elutes at $29 \mathrm{~min}$ contains amino acid residues 126-141 resulting from tryptic cleavage at ${ }^{125} \mathrm{Arg}$ and the minor species that elutes at $30.4 \mathrm{~min}$ contains amino acid residues $127-141$ resulting from cleavage at ${ }^{126}$ Lys.

The full-scan mass spectra of the major glycopeptides corresponding to ${ }^{138} \mathrm{Asn}$ and ${ }^{81} \mathrm{Asn}$ are shown in Figure $5 a$ and $b$, respectively. Each mass spectrum contains the HexHexNAc oxonium ion at $m / z 366$ used to locate the glycopeptides in the proteolytic digest mixture. In Figure 5a the multiply charged

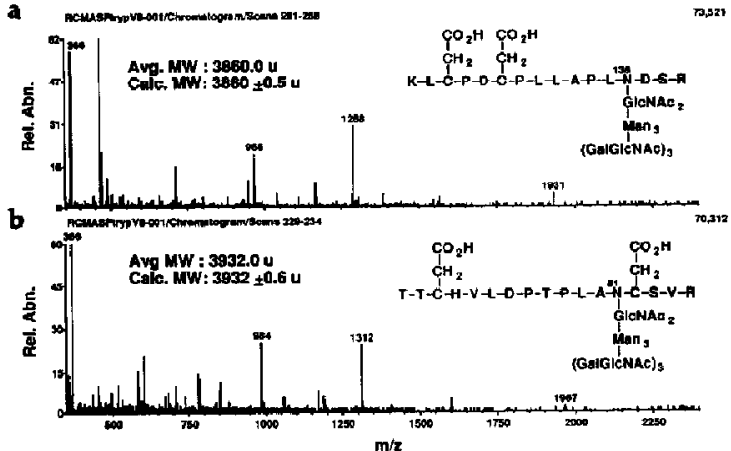

Figure 5. (a) Background-subtracted, averaged mass spectrum of chromatographic peak at 29 min of Figure 4a corresponding to glycopeptides containing ${ }^{138}$ Asn resulting from cleavage at ${ }^{125}$ Arg and ${ }^{141}$ Arg of ASF. (b) Background-subtracted, averaged mass spectrum of chromatographic peak at $23.7 \mathrm{~min}$ of Figure $4 \mathrm{a}$ corresponding to glycopeptides containing ${ }^{81}$ Asn resulting from cleavage at ${ }^{68} \mathrm{Glu}$ and ${ }^{85} \mathrm{Arg}$ of ASF. 
series of ions at $m / z 966,1288$, and 1931 corresponds to the $[\mathrm{M}+4 \mathrm{H}]^{4+},[\mathrm{M}+3 \mathrm{H}]^{3+}$, and $[\mathrm{M}+2 \mathrm{H}]^{2+}$ species of the glycopeptide with the proposed structure shown in the inset. The major $\mathrm{N}$-linked asialooligosaccharide reported to occur in fetuin consists of triantennary sugar chains containing the Gal $\beta 1$ $\rightarrow 3 \mathrm{GlcNAc}$ or Gal $\beta 1 \rightarrow$ 4GlcNAc sequence attached to the trimannosyl chitobiose core [59]. The calculated mass from the multiply charged ions observed in these data agrees with the average mass of $3860 u$ for this reduced and carboxymethylated ${ }^{138} \mathrm{Asn}$ glycopeptide. The unlabeled ions at $m / z 465$ and 709 presumably correspond to a coeluting, unidentified peptide. Extracted ion profiles of these ions and each of the labeled ions (not shown) demonstrated that the latter two components eluted at a slightly later retention time. The full-scan mass spectrum in Figure $5 b$ shows the multiply charged ion series at $m / z$ 984, 1312, and 1967 corresponding to the $[\mathrm{M}+4 \mathrm{H}]^{4+},[\mathrm{M}+3 \mathrm{H}]^{3+}$, and $[\mathrm{M}+2 \mathrm{H}]^{2+}$ species of the ${ }^{81}$ Asn glycopeptide with the proposed structure shown in the inset. Once again, the calculated molecular weight of $3932 \mathrm{u}$ is in close agreement with the average molecular weight of the reduced and carboxymethylated glycopeptide. 5

The sialic acid present in a complex glycopeptide can give rise to oxonium ions at $m / z 292$ and 366 (which correspond to the oxonium ions for NeuNAc and HexHexNAc, respectively). Figure 6a shows the total extracted ion chromatogram for $m / z 292$ and 366 from a full-scan LC/CID/MS analysis of a peptide mixture resulting from the digestion of RCM fetuin with trypsin that was scanned over $m / z$ 200-2400 with a $60-\mathrm{V}$ declustering potential. The replotted total ion chromatogram from $m / z 350$ to 2000 is shown in Figure $6 \mathrm{~b}$. The background-subtracted mass spectrum of the glycopeptide eluting at $38 \mathrm{~min}$ (in Figure 6a) is shown in Figure 7a. The declustering potential of $60 \mathrm{~V}$ was sufficient to produce $\mathrm{HexNAc}$, NeuNAc- $\mathrm{H}_{2} \mathrm{O}$, NeuNAc, HexHexNAc and NeuAcHexHexNAc oxo-

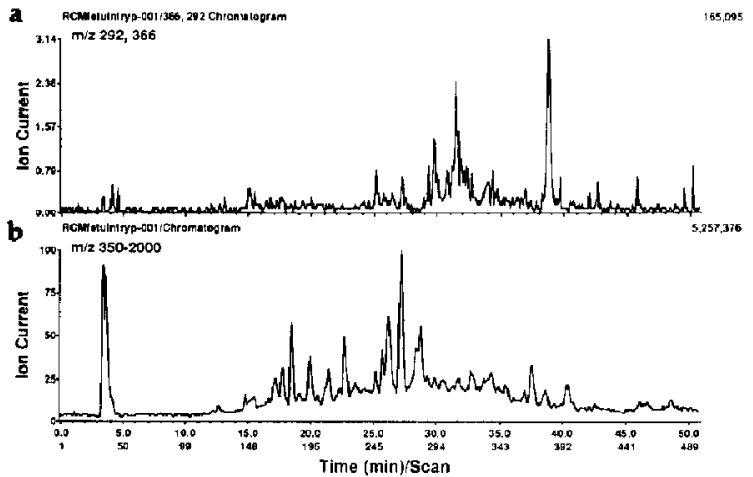

Figure 6. (a) LC/CID/MS total extracted ion chromatogram for $m / z 292$ and 366 resulting from a $20-\mu \mathrm{L}$ injection of RCM fetuin digested with trypsin (ca. 10 nunol injected). Data were acquired by scanning $Q_{1}$ from $m / z 200$ to 2400 in $1.0 \mathrm{u}$ steps for 2.73 $\mathrm{msec} / \mathrm{step}(6.1 \mathrm{sec} / \mathrm{scan})$ with a declustering potential of $60 \mathrm{~V}$. (b) Total ion chromatogram replotted from $m / z 350$ to 2000 . See text for chromatographic conditions.

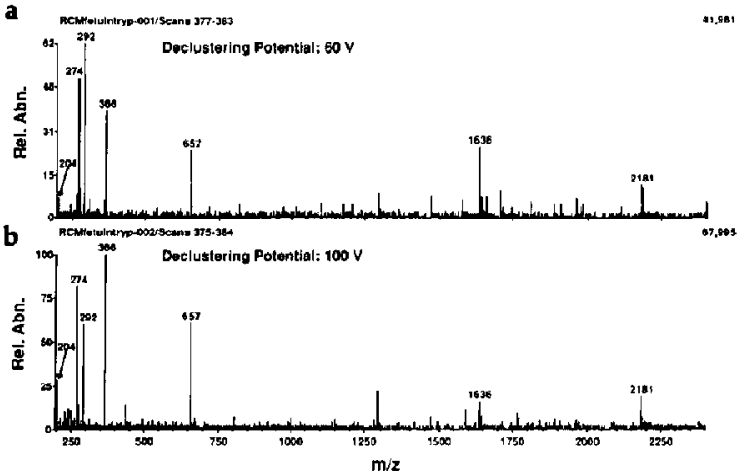

Figure 7. (a) Background-subtracted, averaged mass spectrum of chromatographic peak at $38 \mathrm{~min}$ of chromatogram shown in Figure 6a. (b) Background-subtracted, averaged mass spectrum of same component shown in (a) except that the declustering potential was increased to $100 \mathrm{~V}$ (total ion chromatogram not shown).

nium ions at $m / z 204,272,292,366$, and 657 , respectively. The ions at $m / z 1636$ and 2181 are consistent with the $[\mathrm{M}+4 \mathrm{H}]^{4+}$ and $[\mathrm{M}+3 \mathrm{H}]^{3+}$ of a putative glycopeptide (or a coeluting peptide) of mass $6540 \mathrm{u}$.

By raising the declustering potential to $100 \mathrm{~V}$ the yield of oxonium ions in the declustering region is increased, but the water loss from the oxonium ion of neuraminic acid is also favored $(\mathrm{m} / z$ 274). A LC/CID/MS experiment of selected abundant oxonium species acquired under SIM conditions is shown in Figure 8. A comparison with Figure 6a shows that a much greater signal-to-noise ratio is achieved by the SIM LC./CID/MS approach. The individual selected ion chromatograms can be used to locate glycopeptides by looking for the coincidence of retention time for the chromatographic profiles of these diagnostic ions. A limitation of this approach is that it offers only a chromatographic screening method to locate suspect peptides for further evaluation. Additional mass spectral data and hence structural information of the intact

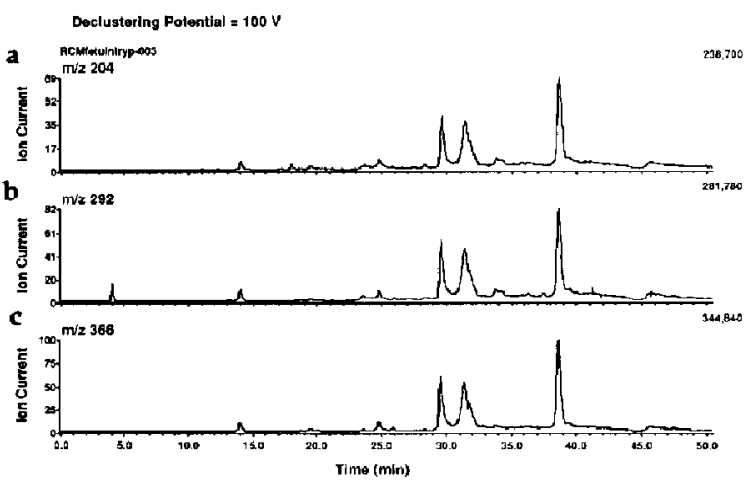

Figure 8. (a) LC/CID/MS selected ion chromatogram of $m / z$ 204 resulting from a $20-\mu \mathrm{L}$ injection of the same sample as in Figure 6 (ca. 10 nmol injected). Data were acquired at $m / z 204$, 292 , and 366 for $500 \mathrm{msec} /$ ion (2.0 sec/cycle) with a declustering potential of $100 \mathrm{~V}$. (b) Selected ion chromatogram of $m / z 292$. (c) Selected ion chromatogram of $m / z$ 366. See text for chromatographic conditions. 
glycopeptides would be required by full-scan LC/ CID/MS or possibly LC/MS/MS.

\section{Conclusions}

Fractionation of enzymatic digest mixtures containing peptides and glycopeptides by solid-phase extraction followed by continuous infusion of the sample into the ion spray interface produces interpretable CID MS /MS mass spectra of the doubly charged glycopeptide precursor ions without the need for chromatographic separation. The mass spectra of these model tryptic glycopeptides adhere to a common template. In the low mass-to-charge ratio region characteristic oxonium ions may be observed. Hex ${ }^{+}\left(m / z\right.$ 163), HexHex ${ }^{+}(m / z$ 325), HexNAc ${ }^{+}\left(m / z\right.$ 204), and HexHexNAc ${ }^{+}(m / z$ 366) are observed with the high-mannose glycopeptides from RNase B. Preferential stabilization by the acetamido group produces only the latter two ions with the complex glycopeptides from ovomucoid. A complete singly charged reducing terminus series with charge retention on the peptide yields sugar composition and partial sequence information; an incomplete, complementary doubly charged ion series is also observed. Usually, the consecutive loss of sugar residues may be followed through the trimannosyl chitobiose core to identify the mass of the original peptide even if the ion is weak or absent. The abundant ion corresponding to the peptide with pendent $\mathrm{N}$-linked GlcNAc and the lack of peptide backbone fragmentation reflects the relative strengths of $\mathrm{O}$ - versus $\mathrm{N}$-linked glycosidic versus anide bonds.

Limitations to this methodology are encountered with the use of a quadrupole analyzer for an inherently high-mass problem. Although tryptic digestion effectively doubles the mass range of the analyzer for the determination of glycoform molecular weights, a higher mass range is required to detect the highest singly charged product ion. The model glycopeptides chosen for the work presented here possess a small tryptic peptide portion to enable the determination of a moderately sized oligosaccharide portion. Many glycoproteins cannot be digested to a conveniently small peptide with trypsin such that other enzymes must be used that may remove the multiply charged nature of the peptide ionization derivative. Complex tetra- and pentaantennary oligosaccharides pose a more challenging structural determination problem than the oligosaccharides used here. Also, the CID fragmentation efficiency decreases as the molecular size increases. Although doubly charged precursor ions facilitate CID to some extent, the use of higher charge state precursor ions may result in charge state ambiguity in the resulting product ions. It is clear that this problem would benefit from the higher mass range, improved mass resolution, and higher collision energies of a magnetic sector instrument [66], Fourier transform mass spectrometry system [67], or ion trap [68, 69] and ongoing efforts to interface these with electrospray should prove beneficial.
The LC/CID/MS approach to screening for glycopeptides involves scanning for glycopeptide ions and oxonium ions produced from them by collisions in the declustering region $\left(\mathrm{Q}_{0}\right)$ of the API triple-quadrupole system. This "up front" CID process involves using only one mass-resolving quadrupole and precludes the scattering losses of ion current and reduced ion transmission efficiency associated with CID MS/MS. Glycopeptides may be located in proteolytic digests of glycoproteins by comparing the extracted ion chromatograms of a variety of oxonium ions $(\mathrm{m} / \mathrm{z}$ $163,204,366$ ) to the total ion chromatogram from data obtained with a single LC/CID/MS experiment. A limitation of this approach is that the limited separation efficiency of HPLC may result in mixed mass spectra that must be background subtracted to obtain a good mass spectrum of the glycopeptide peak. Thus the ions in the resulting mass spectrum must be assigned to either related glycoforms, coeluting peptides, or fragment ions. Also, the distribution of glycoform ion intensities will be affected by the collisional processes in the declustering region and therefore will not necessarily represent the glycosylation of the native glycoprotein. A possible solution would be a massdependent scan of the orifice potential to produce oxonium ions with high declustering potential at low mass and intact glycopeptides with low declustering potential at higher mass. Improved software with the Sciex instrument should make these experiments possible.

Finally, SIM LC/CID/MS determination of the characteristic oxonium ions produced in the declustering region provides an even greater signal-to-noise response than full-scan LC/CID/MS for the detection of glycopeptides. If the LC/MS system is incorporated with a postcolumn split [70], then the putative glycopeptides can be isolated and further characterized by additional experiments. The lack of full-scan mass spectral information limits the utility of SIM LC/ CID/MS to one that only screens HPLC peptide maps for possible glycopeptides. It may be a useful alternative, however, as a first experiment when sample quantities are limited.

\section{Acknowledgments}

The authors would like to express their thanks to PE-Sciex, Inc. for financial and hardware support, Applied Biosystems, Inc. for the donation of the HPLC micropump, Dr. T. G. Huggins for technical assistance with proteolytic digests, and Drs. S. A. Carr and T. R. Covey for many helpful discussions.

\section{References}

1. Carr, S. A.; Hemling, M. E.; Bean, M. F,; Roberts, G. D. Anal. Chem. 1991, 63, 2802-2824.

2. Spellman, M. W. Anal. Chem. 1990, 62, 1714-1722.

3. Rademacher, T. W.; Parekh, R. B.; Dwek, R. A. Annu. Rev. Biochem. 1988, 57, 785-838.

4. Merkle, R. K; Cummings, R. D. In Methods in Enzymology, Complex Carbohydrates (Part E), Vol. 138; Ginsburg, V., Ed:; Academic: New York, 1987; pp 232-259. 
5. Yamashita, K.; Mizuochi, T.; Kobata, A. In Methods in Enzymology, Complex Carbohydrates (Part D), Vol. 83; Ginsburg, V., Ed; Academic: New York, 1982; pp 105-126.

6. Lindberg, B. In Methods in Enzymology, Complex Carbohydrates (Part B), Vol. 28; Ginsburg, V., Ed.; Academic: New York, 1972; pp 178-195.

7. Dell, A. Adv, Carbohydr. Chem. Biochem. 1987, 45, 19-72.

8. Egge, H.; Peter-Katalinic, J. Mass Spectrom. Ret. 1987, 6, 331-393.

9. Barber, M.; Borduli, R. S.; Sedgwick, R. D.; Tyler, A. N. J. Chem. Soc. Chem. Comm. 1981, 325-327.

10. Dell, A. In Methods in Enzymology, Vol. 193; McCloskey, J. A., Ed.: Academic: San Diego, 1990; pp 647-660.

11. Domon, B, Costello, C. E. Glycoconjugate ). 1988, 5, 397-409.

12. Poulter, L.; Burlingame, A. L. In Methods in Enzymology, Vol. 193; McCloskey, J. A., Ed.; Academic: San Diego, 1990; Pp 661-689.

13. Gillece-Castro, B. L.; Burlingame, A. L. In Methods in Enzymology, Vol. 193; McCloskey, J. A., Ed; Academic: San Diego, 1990; pp 689712.

14. Carr, S. A.; Barr, J. R.; Ruberts, G. D.; Anumula, K. R.; Taylor, P. B. In Methods in Enzymology, Vol. 193; McCloskey, J. A., Ed.; Academic: San Diego, 1990; pp 501-518.

15. Carr, S. A.; Roberts, G. D. Anal. Biochem. 1986, 157, 396-406.

16. Carr, S. A.; Hemling, M. E.; Folena-Wasserman, G.; Sweet, R. W.; Annumula, K.; Barr, J. R.; Huddleston, M. J.; Taylor, P. J. Biol. Chem. 1989, 264, 21286-21245.

17. Struck, D. K., Lennarz, W. J. In The Biochemistry of Glycoproteins and Proteoglycans; Lennarz, W. J., Ed.; Plenum: New York, 1980; $p 35$.

18. Fenn, J. B.; Mann, M.; Meng, C. K.; Wong, S. F.; Whitehouse, C. M. Science 1989, 246, 64-71.

19. Iribarne, J. V.; Dziedzic, P. J.; Thomson, B. A. Int. J. Mass Spectrom. Ion Phys. 1983, 50,331-347.

20. Naylor, S.; Findeis, F.; Gibson, B. W.; Williams, D. H. J. Am. Chem. Soc. 1986, 108, 6359-6363.

21. Schneider, R. P.; Lynch, M. J.; Ericson, J. F.; Fouda, H. G. Anal. Chem. 1991, 63, 1789-1794.

22. Duffin, K. L.; Henion, J. D.; Huang. E. C. Anal. Chem. 1992, $64,1440-1448$

23. Tsarbopoulos, A.; Her, G. R.; Lam, Z.; Reinhold, B.; Reinhold, V.; Karas, M.; Strupat, K.; Hillencamp, F.; Pramanik, B. N. Proceedings of 39th ASMS Conference on Mass Spectrometry and Allied Topics; 1991; pp 488-489.

24. Reinhold, B,; Reinhold, V. Proceeding of 39th ASMS Conference on Mass Spectrometry and Allied Topics; 1991; pp 232-233.

25. Hunl, D. F.; Zluu, N. Z; Shabanowitz, J. Rapid Commun. Mass Spectrom. 1989, 3, 122-124.

26. Covey, T. R.; Huang, E. C.; Henion, J. D. Anal. Chem. 1991, 63, $1193-1200$.

27. Huang, E. H.; Wachs, T.; Conboy, J. J.; Henion, J. D. Anal. Chem. 1990, 62, 713A-725A

28. Bruins, A. P.; Covey, T. R.; Henion, J. D. Anal. Chem. 1987, $59,2642-2646$.

29. Huang, E. C.; Henion, J. D. I. Am. Soc. Mass Spectrom. 1990, 1, 158-165.

30. Huang, E. C.; Henion, J. D. Anal. Chem 1991, 63, 73?-739.

31. Dawson, P. H.; French, J. B.; Buckley, J. A.; Douglas, D. J.; Simmons, D. Org. Mass Spectrom. 1982, 17, 205-211.

32. Thannhauser, T. W.; Konishi, Y.; Scheraga, H. A. In Methods in Enzymology. Vol. 143; Jakoby, W. B.; Griffith, O., Eds.; Academic: New York, 1987; pp 115-119.

33. Thannhauser, T, W.; McWherter, C. A,; Scheraga, H. A. Anal. Biochem. 1985, 149, 322-330.

34. Crestfield, A. M.; Moore, S.; Stein, W. H. I. Biol. Chem. 1963, $238,622-627$.

35. Bruins, A. P.; Weidolf, L. O. G.; Henion, J. D. Anal. Chem 1987, 59, 2647-2652.

36. Conboy, J. J.; Henion, J. D.; Martin, M. W.; Zweigenbaum, J. A. Anal. Chem. 1990, 62, 800-807
37. Hunt $r$ D. F.; Yates, J. R.; Shabanowitz, J.; Winston, S.; Hauer, C. R. Proc. Natl. Acad. Sci. 1986, 6233-6237.

38. Covey, T. R.; Bonner, R. F.; Sushan, B. I.; Henion, J. D. Rapid Commun. Mass Spectrom. 1988, 2, 249-255.

39. Loo, J. A.; Edmonds, C. G.; Smith, R. D. Anal. Chem. 1991, 63, $2488-2499$.

40. Yergey, J.; Heller, D.; Hansen, G.; Cotter, R. J.; Fenselau, C. Anal. Chem. 1983, 55, 535-536.

41. Carr, S. A.; Reinhold, V. N.; Green, B. N.; Hass, J. R. Biomed. Mass Spectrom. 1985, 12, 288-295.

42. Beeley, J. G. Biochem. I. 1976, 159, 335-345

43. Yet, M. G. Chin, C. C. Q.; Wold, F. J. Biol. Chem. 1988, 263, 111-117.

44. Yamashita, K.; Kamerling, J. P.: Kobata, A. I. Biol. Chem. 1982, 257, 12809-12814.

45. Yamashita, K.; Kamerling, J. P.; Kobata, A. I. Biol. Chem. 1983, 258, 3099-3106.

46. Bjorndal, H.; Hellerqvist, C. G.; Lindberg, B.; Svensson, 5. Angew. Chem. Int. Ed. Engl. 1970, 9, 610-619.

47. Dawson, P. H.; French, J. B.; Buckley, J. A.; Douglas, D. J.; Simmons, D. Org. Mass Spectrom. 1982, 17, 212-219.

48. API III LC/MS/MS Operator's Manual (009658-C); Sciex, Thorrhill, Ontario, June 1991; B7-B8.

49. Tai, T; Yamashita, K.; Kobata, A. Biochem. Biophys. Res. Commur. 1977, 78, 434-441.

50. Huddleston, M. J.; Bean, M. F.; Barr, J. R.; Carr, S. A Proceedings of the 39th ASMS Conference on Mass Spectrometry and Allied Topics; 1991; p. 280-281.

51. Covey, T, R. Ph.D. Thesis, Comell University, January, 1987.

52. Katta, V.; Chowdury, S. K.; Chait, B. T. Anal Chem 1991, 63, 174-178.

53. Allen, M. H.; Vestal, M. L. J. Am. Soc. Mass Spectrom. 1992, $3,18-26$

54. Smith, R. D; Loo, J. A.; Barinaga, C. J.; Edmonds, C. G.; Udseth, H. R. J. Am. Soc. Mass Spectrom. 1990, 1, 53-65.

55. Meng, C. K.; McEwen, C. N.; Larsen, B. S. Rapid Commun. Mass Spedrom. 1990, 4, 151-155.

56. Dixon, M.; Webb, E. C. Enzymes, 2nd ed.; Academic: New York, 1964; Chapter 6. pp 243-245.

57. Bruins. A. P. Mass Spectrom. Rev. 1991, 10, 53-77.

58. Dziegielewska, K. M.; Brown, W. M.; Casey, S.; Christie, D. L.; Foreman, R. C.; Hill, R. M.; Saunders, N. R. J. Btol. Chem. 1989, 265, 4354-4357.

59. Spiro, R. G.; Bhoyroo, V. D. I. Biol. Chem. 1974, 249, 5704-5717.

60. Nilsson, B.; Norden, N. E.; Svensson, S. J. Biol. Chem. 1979, $254,4545-4553$.

61. Baenziger, J. U.; Fiete, D. I. Biol. Chem. 1979, 254, 789-795.

62. Takasaki, S.; Kobata, A. Biochem. 1986, 25, 5709-5715.

63. Townsend, R. R.; Hardy, H. R.; Wong, T. C.; Lee, Y. C. Biochem. 1986, 25, 5716-5725.

64. Bendiak, B.; Harris-Brandts, M.; Michnik, S. W.; Carver, J. P.; Cumming, D. A. Biochem. 1989, 28,6491-6499.

65. Cumming, D. A; Hellerquist, C. G.; Harris-Brandts, M.; Michnik, S. W.; Carver, J. P.; Bendiak, B. Biochem. 1989, 28, 6500-6512.

66. Larsen, B. S.; McEwen, C. N. J. Am. Soc. Mass Spectrom. 1991, $2,205-211$

67. Henry, K. D; Williams, E. R; Wang, B. H; McLafferty, F.W.; Shabanowitz, J.; Hunt, D. F. Proc. Natl. Acad. Sci. USA 1989, $86,9075-9078$.

68. Cooks, R. G.; Cox, K.; Williams, J. D.; Morand, K. L.; Julian, R. K.; Kaiser, R. E. Proceedings of the 39th Conference on Mass Spectrometry and Allied Topics; 1991; pp 469-470.

69. Williams, J. D.; Cox, K.; Morand, K. L.; Cooks, R .G.; Julian, R. K.; Kaiser, R .E. Proceedings of the 39 th Conference on Mass Spectrometry and Allied Topics; 1991; pp 1481-1482.

70. Ling, V.; Guzzetta, A. W.; Canova-Davis, E.; Stults, J. T.; Hancock, W. S.; Covey, T. R.; Shushan, B. I. Anal. Chem. 1991, 63, 2909-2915. 